\title{
Isomerismo atrial esquerdo no defeito do septo atrioventricular parcial
}

\section{Left atrial isomerism in the defect of partial atrioventricular septum}

Ulisses Alexandre CROTI, Domingo Marcolino BRAILE, Sírio HASSEM SOBRINHO, Moacir Fernandes de GODOY

\section{DADOS CLIÍNICOS}

Criança de 2 anos, sexo feminino, parda, natural da Bahia. Apresentava cansaço às mamadas desde o nascimento, o qual melhorou com uso de digital e diuréticos. REG, descorada, dispnéia leve. Ictus cordis palpável no $5^{\circ}$ espaço intercostal à esquerda, ritmo cardíaco regular em dois tempos, sopro sistólico $+4 / 6+$ em bordo esternal esquerdo médio e hiperfonese de segunda bulha. Ausculta pulmonar normal. Abdome com fígado a $3 \mathrm{~cm}$ do RCD. Pulsos presentes e palpáveis nos quatro membros.

\section{ELETROCARDIOGRAMA}

Ritmo atrial direito baixo, freqüência de 115 bpm. SÂP -60, SÂQRS indeterminado, intervalo PR 0,12s, QRS 0,10s e QTc 0,38 s. Sobrecarga ventricular direita evidenciada por complexos qR em V1 e RS em V6 (Figura 1).

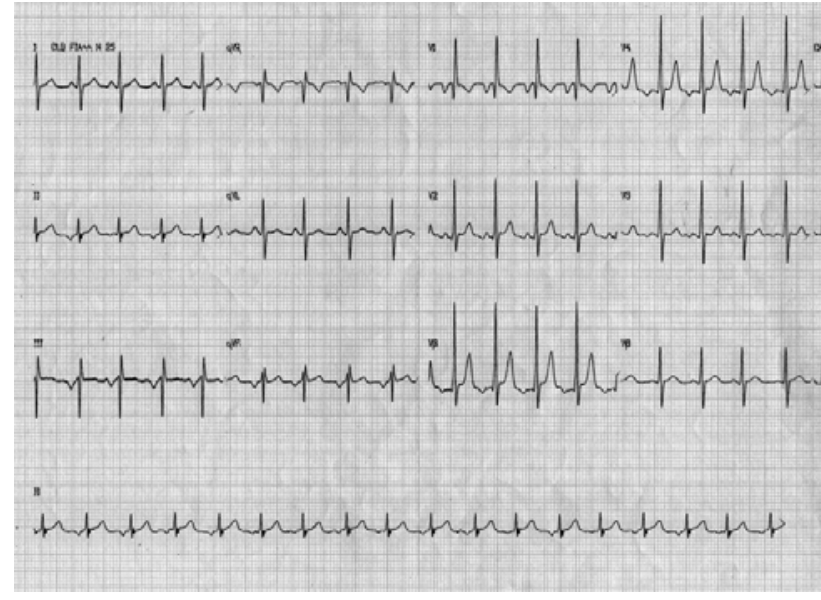

Fig. 1 - Eletrocardiograma pré-operatório

Trabalho realizado no Serviço de Cirurgia Cardiovascular Pediátrica de São José do Rio Preto - Hospital de Base - Faculdade de Medicina de São José do Rio Preto.

Endereço para correspondência: Ulisses Alexandre Croti Hospital de Base - FAMERP - Avenida Brigadeiro Faria Lima, 5544 CEP 15090-000 - São José do Rio Preto - São Paulo Fone (Fax): 17 - 32015025 / 32226450 / 97726560 E-mail: uacroti@uol.com.br 


\section{RADIOGRAMA}

Situs solitus visceral. Área cardíaca aumentada com ICT 0,64 . Proeminência da trama vascular tipo shunt, redução volumétrica do lobo inferior esquerdo. Cúpula diafragmática livre à direita.

\section{ECOCARDIOGRAMA}

Situs solitus em levocardia. Conexão venoatrial, atrioventricular e ventriculoarterial concordante. Defeito do septo atrioventricular forma parcial, isomerismo atrial esquerdo, insuficiência das valvas atrioventriculares, sendo à esquerda de grau importante e à direita, discreto. Artéria pulmonar esquerda com $10 \mathrm{~mm}$ e direita com 6,5 $\mathrm{mm}$ na origem e $10 \mathrm{~mm}$ distalmente, apresentando gradiente ao Doppler de 58 mmHg. Relação Qp/Qs estimada em 4.

\section{DIAGNÓSTICO}

A história clínica de insuficiência cardíaca congestiva com hiperfonese de segunda bulha orienta para cardiopatia congênita de hiperfluxo pulmonar e hipertensão pulmonar. O ecocardiograma determinou as características anatômicas com precisão e, diante do achado de isomerismo atrial esquerdo, foi atentado ao posicionamento adequado dos músculos papilares, já que havia presença de insuficiência valvar importante, para adequada correção cirúrgica [1]. Também foi realizada ultra-sonografia abdominal com intuito de excluir presença de poliesplenia.

\section{OPERAÇÃO}

Toracotomia transesternal mediana, instalação do auxílio da circulação extracorpórea, hipotermia a $28^{\circ} \mathrm{C}$, cardioplegia sanguínea anterógrada, intermitente, hipotérmica, repetida a cada 20 minutos. Abertura de átrio morfologicamente esquerdo à direita (Figura 2) e tronco pulmonar. Não visibilizada estenose anatômica na origem da artéria pulmonar direita, porém optado por ampliação do terço inicial com placa de pericárdio autólogo devido à possibilidade de estenose funcional demonstrada previamente ao ecocardiograma. A fenda da valva atrioventricular esquerda foi fechada com pontos separados de polipropileno 6-0. A comunicação interatrial tipo ostium primum foi fechada com

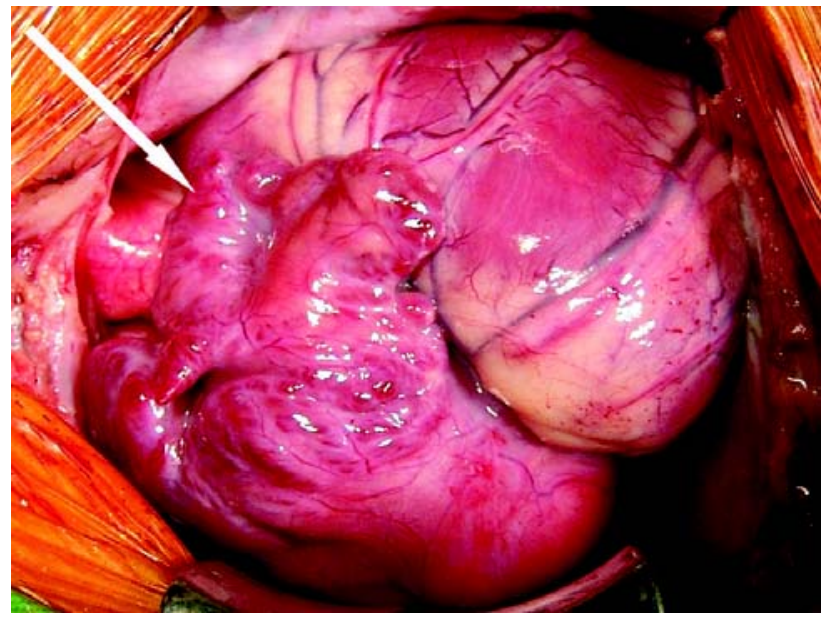

Fig. 2 - Isomerismo atrial esquerdo (seta)

placa de pericárdio bovino com sutura contínua, também de polipropileno 6-0, deixando o seio coronário para a direita. O tempo de perfusão foi de 61 minutos e de isquemia miocárdica, de 48 minutos. Não houve dificuldades no tratamento da valva atrioventricular esquerda e, ao teste com solução salina, apresentava-se competente. No $4^{\circ}$ dia de pós-operatório, na enfermaria, apresentou parada cardiorrespiratória por aspiração de leite. Após este evento, evoluiu satisfatoriamente do ponto de vista cardiovascular, com ecocardiograma de controle demonstrando correção do defeito com regurgitação trivial pela valva atrioventricular esquerda e diminuição importante do tamanho da área cardíaca, porém recebeu alta hospitalar com grave seqüela neurológica.

\section{REFERÊNCIA}

1. Uemura H, Anderson RH, Ho SY, Devine WA, Neches WH, Smith A, et al. Left ventricular structures in atrioventricular septal defect associated with isomerism of atrial appendages compared with similar features with usual atrial arrangement. J Thorac Cardiovasc Surg. 1995;110(2):436-44. 